\title{
Three Dimensional Agricultural Land Modeling using Unmanned Aerial System (UAS)
}

\author{
Faisal Mahmood ${ }^{1}$, Khizar Abbas ${ }^{2}$, Asif Raza ${ }^{3}$, Muhammad Awais Khan ${ }^{4}$, Prince Waqas Khan ${ }^{5}$ \\ Department of Computer Science \\ University of Agriculture Faisalabad, Pakistan 1,2,5 \\ Bahauddin Zakariya University, Multan, Pakistan ${ }^{3,4}$
}

\begin{abstract}
Nowadays, the unmanned aerial vehicles (UAVs) drones are mostly used in civil and military fields for security and monitoring purposes. They are also involved in the development of electronics communications and navigation systems. The UAVs are the aerial vehicles with a built-in power system having capability of controlling by a remote control system or leads to fly automatically. Rapid increase in their use due to sensors mobility in its small size that becomes the UAVs to fly at lower altitude and their significant contributions to the image processing studies, where the photogrammetric surveys in small scale areas are given importance for landslide and erosion monitoring. This paper is going to consider agriculture activities like detecting crop diseases, finding crop patterns and conduct small scale agriculture policies for study and research. In our study, the UAV drone is used for the image data collection purpose and structure form motion (SfM), algorithmic approach is utilized for producing the volumetric structure or 3-D structure of images. These 3-dimensional structures are further used for building information modeling systems and performing different operations like image classification, enhancement and segmentation. Our approach highlights better and efficient results than others agriculture images approaches captured by UAVs at high altitude.
\end{abstract}

Keywords-Image processing; structure from motion (SFM); unmanned aerial system (UAS); unmanned aerial vehicles (UAVs); camera calibration; change detection

\section{INTRODUCTION}

\section{A. Unmanned Ariel System Photogrammetry}

From a short period of time, the presentation of Digital Photogrammetry methods has been improved expressively that is used for accumulating cartographic information's from pictures extract by ordinal cameras included in Unmanned Aerial System (UAS). The appearance of UAS equipment's can be recognized by procedural progresses of electrical mechanisms and the opportunities in those aircrafts that are controlled by remote. UAS is mostly found in Artificial Intelligence, Computer Science and Engineering and also be used in the Photogrammetry as well as in Remote Sensing populations. "UAS photogrammetry" defines a photogrammetric dimension policy which drives slightly controlled vehicles without pilot. Unmanned Aerial System (UAS) give a promising and versatile stage for the securing of multi-short lived Digital surface models (DSMs) and ortho rectified air images [1]. Unmanned Aerial System (UAS) are a stimulating new remote identifying gadget fit for increasing high assurance spatial data UAS systems process releases many other latest applications in the adjacent variety field, relating airborne and global photogrammetry. It is a latest claim and low-cost substitutes to the typical operated aerial photogrammetry [13]. Foremost structures of UAS photogrammetry are deliberated with the estimation of costs (low-cost), brief elevation (low-high), ability of picture achievement in actual time for example worth based on device structures flight performance, impact on impressive and surrounding environment, windflaws etc. some other types or groups of UAS are also useful for photogrammetric data achievement. Expressed that our test augment has shown that the capability of UAS photogrammetry interestingly with other estimation propels depends on upon a couple of parts, for instance, the measure of the area of interest, which in this manner impacts the amount of included pictures.

In this research paper the UAS photogrammetry is utilized and a UAV quad captor is used for capturing the images from high altitude with four mounted cameras. The University of Agriculture Pakistan (UAF) cultivated land is used for this photography. After collecting the images data from quad copter the image preprocessing actions are perform for cleaning these high resolution images. The distortion removal operation is also performed at collected data. The structure form motion (SfM) techniques are applied for the construction of 3-D models. After that's these 3-D models are further utilized for developing business information system and also other image processing tasks like image classification, segmentation and enhancement. The steps of research work are shown in Fig. 1.

Using specialized digital photogrammetric cameras consents produce high feature digital surface models (DSM) with picture identical. This type of external data is significant for producing 3D structure replicas, landscape imagining, top figures, shade models, etc. and update conventional digital landscape models [10]. The invention of orthophoto as well as digital elevation models (DEMs) turn to entirely ordinal and by less output time. These key features guaranteeing in attainment of cartographic data by ordinal images. Ordinal photogrammetry procedures are useful to make an orthophoto graphic maps.

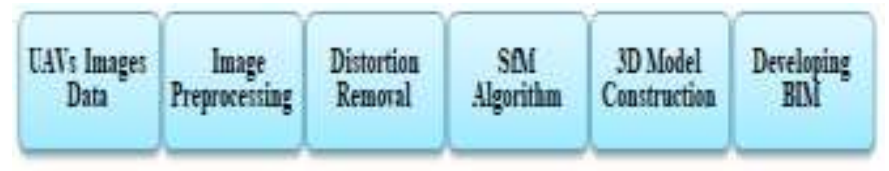

Fig. 1. Research Process. 


\section{B. Structure from Motion (SfM)}

Here study, we bang on a developing, less-price photogrammetric technique for better determination topographic reestablishment, perfectly right for low-priced investigation and use in distant regions. SfM functions under the same simple principles for example stereoscopic photogrammetry that is that 3-D arrangement can be determined from a sequence of intersecting, equalizer images (Fig. 1). Though, it varies basically from straight photogrammetry, in that the geometry of the act, camera sites and alignment is resolved mechanically lacking the basic near identify a priori, a system of objectives which have known 3D locations. In its place, these are resolved instantaneously by a highly terminated, iterative bundle change process, built on a file of types mechanically removed from a regular of many intersecting pictures [15]. As defined below (Fig. 2), the method is best fit to groups of pictures with a great grade of intersection that arrest complete three-D arrangement of the act observed from extensive collection of sites, or as the name proposes, images resultant from an affecting device.

Instead of a single stereo pair, the SfM method wants multiple, overlapping points as input to feature abstraction and 3 -D reconstruction systems. Explained the progressions in the remote identifying of fluvial structures have given investigators uncommon points of view on the versatile nature of conduits [2]. An aeronautical perspective is basic to mapping and understanding the stream at an arrangement of spatial scales. This simplicity course of action conveyed high spatial assurance airborne photography and electronic ascent models for a 32-km area of the Middle Fork John Day River in east central Oregon.

This method was established in the 1990s and has its roots in the computer image municipal and the progress of programmed feature-corresponding systems in the earlier time. The method has been promoted over a variety of hazehandling devices, most especially Microsoft Photosynth which uses SfM methods recognized in 2008. These apparatuses can mark straight usage of customer-uploaded and pack found taking pictures to produce the essential treatment of an objective act, and can mechanically produce thin 3-D point clouds from these photosets [4]. The opportunities of SfM perform endless, though, to era, the method has hardly been used inside the geosciences and there occur few measurable calculations of the quality of environment yields resultant from this method [12].

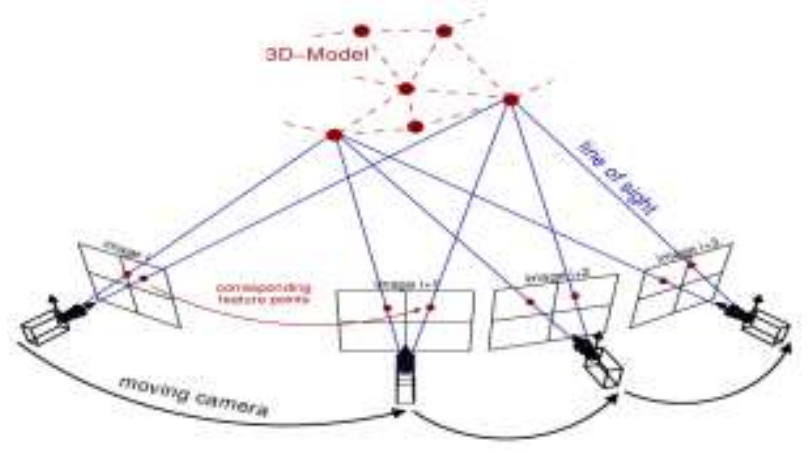

Fig. 2. Structure-from-Motion (SfM) [4].

\section{Land Modelling in Agriculture}

Agricultural determination planning trainings accepted out through traditional procedures which entire situation, GPS, laser scanners, digital cameras and other tools are being produced and used in as built generally on close-range assessing systems. Even though accuracy is great in this way; workforce, time and cost are aggregate and in the same time, it affects accuracy to become lower most of the time in evaluating of facts which are hard to be achieved to or some facts can't be restrained at all.

UAS Photogrammetry certainly releases many new submissions in the close-range photogrammetry in the geomantic field. Drawing generation by UAS methods is a combined method between earth systems and aerial plot group systems. All assessing tools demanding point gaining are being incorporated into UAS flying at low elevation as unlike from satellites or airplanes. All 3-D data are being executed aerially and carefully without crushed control ideas. The Paper is divided into five sections. In the first section introduction of the paper and main idea is explained. The second section is related to background literature and third section is related to the material and methods. The fourth section is result and discussion and at the last conclusion of the paper is discussed.

\section{BACKGROUND AND LITERATURE REVIEW}

SfM counts immense support in the era of basic essential land models from images assembled using unmanned aerial System (UAS). Regardless, the review quality finished in appropriated geomorphological examinations is significantly factor, and satisfactory get ready purposes of intrigue are never given to see totally the explanations behind variability. In both logical examinations, the Monte Carlo approach gave an energetic demonstrate that field effort could by essentially reduced by simply sending about an expansive part of the amount of GCPs, with immaterial effect on the investigation quality. To diminish get ready old rarities and propel confide in SfM-based geomorphological reviews, disseminated results should fuse taking care of unpretentious components which fuse the photo residuals for both tie centers and GCPs, and assurance that these are considered fittingly inside the work procedure [5].

The proposed system is remote identifying has been supporting ancient investigations since the mid twentieth century. Late high-assurance satellites have engaged the quick and dirty observation and mapping over wide domains enhancing the hugeness of remote recognizing strategies. The accuracy of the orthorectified picture was avowed by evaluating the root suggests square goof [14]. The parallel bank like parts of the Tangshan complex were reproduced and their sensible limit prescribed. The straightforwardness of the SfM planning is particularly precious when dealing with CORONA satellite pictures, since it doesn't require the camera parameters, which are frequently unverifiable. The orthophotos gotten from the CORONA satellite pictures were comparatively exact to standard systems. Regardless, the derived shape maps were defiled by spot fuss.

It stated that the paper shows the work on fusing an Advanced Surface Movement Guidance and Control system 
with warm imaging based block acknowledgment and advised structure. The fact of the matter is to engage later on UAS to work accommodatingly with watched out for flying machine and enable independent moving of UAS in possessed and complex plane terminal focus focuses. The A-SMGCS system relied upon Frankfurt air terminal guide in Germany, while the warm imaging used to develop the vehicle area figuring were taken at Cranfield plane terminal in UK [11]. The work shown in this paper shortens the hidden test results gotten for working up an Advanced Surface Movement and Obstacle Detection system for unmanned vehicles. The chairman would pre-outline the taxi course, including all the hold centers till the edge of the runway.

They proposed that we offered a solid and modified work handle which can agreement with the way that ultra-light UAS give only by and large off course information about the position and presentation of the got pictures. This confined precision would generally speak to an issue to for ordinary photogrammetric work forms which want a significant measure of physical work to finish comes to fruition. The showed post taking care of make use of later and capable PC vision techniques to beat this issue [6]. This can be astoundingly useful in many arenas, for instance, cultivating, arrive organization, officer benefit, magnanimous guide, mission masterminding, mining, outline, bygone investigation, urban orchestrating, topography, untamed life watching, officer benefit and various others.

This work describe that ancient examinations are benefitting from fresh mechanical enhancements that are showing new recording systems develop essentially as for 3D showing. Innovative mechanized recordings are upgrading key parts of archeological work on, including precision and adequacy. This is the circumstance of a novel philosophy that uses Unmanned Aerial System (UAS) for data obtainment and programming, for instance, Structure from Motion (SfM) to convey volumetric models from photographs. These photorealistic 3D models can be arranged further using Building Information Modeling (BIM) to make outlines, ranges, electronic ascent models, orthophotographs and diverse sorts of pictures profitable for examination and appropriation [3]. Propelled developments are changing the way ancient investigations is practiced by improving a persistently broadening set of recording, efficient and dispersal devices [7]. From among the particular 3D showing techniques, Structure from Motion (SfM) has starting late created as another framework that gives better results and a higher assurance than standard systems, for instance, plan drawing and photogrammetry or a bit of the forefront recording strategies including laser inspecting and LiDAR.

\section{MAterial AND Methodology}

In the midst of a singular self-administering photogrammetric flight, the camera, mounted on the UAS stage, can often in gradual countless. The free flight uses the autopilot stack up and PC programming Mission Planner. It uses a ready to manage (e.g., from Google Earth), which is required for planning of the flight, to be particular by indicating destination. It helps controlling self-representing initiating of pictures and self-overseeing take-off and landing. the Quad Copter UAS is a generous, delicacy exertion, low weight UAS plat-shape with foam advancement, a wingspan of $1.8 \mathrm{~m}$ and a weight of around $2 \mathrm{~kg}$. Its speed is around 14 $\mathrm{m} / \mathrm{s}$. It can fly up to $30 \mathrm{~min}$ on low wind conditions. In this way, the most outrageous flight partition is approx. $25 \mathrm{~km}$, considering imperativeness for climbing and landing. The flying stature can be picked in the range from $150 \mathrm{~m}$ to $300 \mathrm{~m}$, depending on the required picture assurance.

The UAS arrange course is totally customized, semimanual or manual. Take-off and touching base on level surface is modified or physical. Auto pilot Ardor Pilot Mega (APM) is used for modified course. It relies on upon the Arduino introduced system. The flight organizing program Mission Planner grants clear and brisk bearing of the motorized fly [9].

Starting now indicated, images increased flying UAS stages give supportive information for different applications, for instance, prehistory documentation, urban zone showing and watching, emergency assessment, and what not [8]. The regular required things are thick point fogs, polygonal models, or ortho pictures which are thusly used for mapping, volume computation, evacuating examinations, representation, city showing, framework, and so on.

\section{A. Camera Parameter}

The camera calibration is divided within two parts internal camera calibration and external camera calibration. The external camera calibration is done by balancing the sensor position to a specific coordinate system. The internal and external camera calibration is also known as the internal parameters and external parameters of the camera. The camera sensor captures multiple images of the same scene and form the calibration matrix and check that the variation in sample images. In this research work, the internal camera parameters are known and we just concentrate on the extrinsic parameters calibration. For external camera calibration, our suggested technique shows that the conversion between the laser coordinate system to camera system identification. This technique is applied to view plan calibrated structure captured with laser finder and camera. This approach limits the external parameters by fixing the laser point in the plan structure to the calculated plan structure from the camera image.

The working of laser scanner system is based on the specific bar or pointer patterns which are mostly seen by the camera. The camera calibration is very important task for vision based systems like laser scanner systems, etc. The conjunction between the camera and laser scanner estimation is very challenging work for the creation of three dimensional structures. The calibration technique is the best solution for estimating this problem and makes the pointer of the laser more visible. We used laser range finder and camera extrinsic calibration whether the bar or laser pointer are unseen in this area [5]. The method of calibration is used in many sensor areas like robotics, flying drones, etc.

Camera calibration was able using the mat lab software. By means of the color-coded goals (Fig. 3) for programmed extents, focal length $\mathrm{c}$, the principal point $\mathrm{x}, \mathrm{y}$ and the radial lens alteration factors were projected within a bundle 
correction. More limitations of lens alteration were not important, so they were ignored. This direct way takes about 1 hour containing image achievement and handling. Camera calibration was shown once before the flight, such that likely variations of the factors cannot be evaluate.

1) Performance w.r.t. the number of checkerboard poses: In this example we captured the different checker board images from different angels (Fig. 4). The experiment show that if used more pose images provide better accuracy than less images. The high amount of different pose images are utilized show less error in the projected plan.

2) Intrinsic parameter: After the experiment, we find the intrinsic parameter of camera that we have used in our image capturing experiment. These parameters are used in the process of structure from motion. Camera calibration app is used for finding these intrinsic and extrinsic parameters of the camera [16] (Table 1).

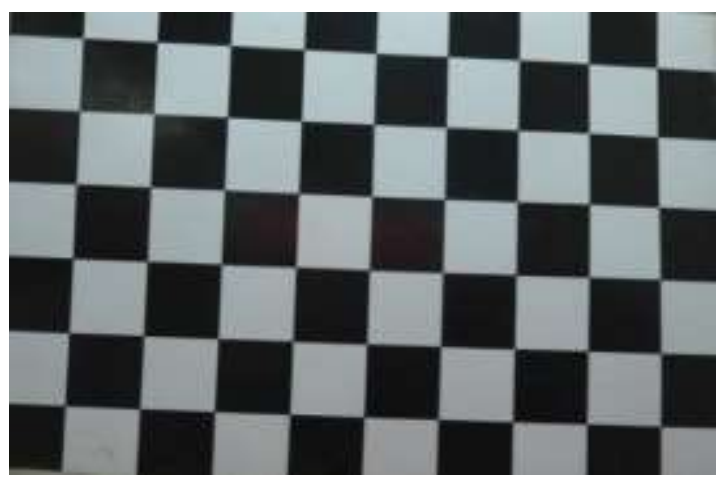

Fig. 3. Checker Board Image of the Calibration for Finding the Camera Parameter [16].

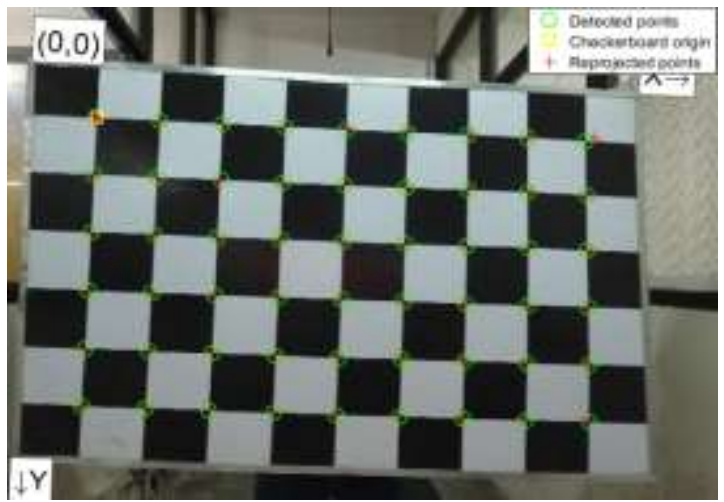

Fig. 4. Detecting and Rejecting Point from Checkerboard Image [16].

TABLE I. INTRINSIC PARAMETERS OF THE CAMERA

\begin{tabular}{|l|l|}
\hline K1 & $1.18189 \mathrm{e}-3$ \\
\hline K2 & $1.65561 \mathrm{e}-5$ \\
\hline K3 & $-1.85267 \mathrm{e}-6$ \\
\hline $\mathbf{P}_{\mathbf{X}}(\mathbf{m m})$ & 0.0593 \\
\hline $\mathbf{P}_{\mathbf{Y}}(\mathbf{m m})$ & -0.0655 \\
\hline Pixel Size $(\mathbf{m m})$ & .0032 \\
\hline
\end{tabular}

\section{RESULTS AND DISCUSSION}

In this research work, the agricultural land area and three dimensional images area are briefly explained in this section. The experimental outcomes show that 3D structure gives accurate calculation of the agriculture land area for different shapes and objects. Many of the freeware tools are used for creating 3D structure but these tools are not efficient enough. We used MATLAB software tool for implementing this technique and no one special or costly hardware is required. e.g. the system specification used in this research 64 bit system, $2.5 \mathrm{GHz}$, RAM $8 \mathrm{~GB}$ with $512 \mathrm{GPU}$ and processing time 5 to $45 \mathrm{~h}$ with 800 images of high resolution.

1) Structure from motion for two views: Structure from motion (SfM) is the process of estimating the 3-D structure of a scene from a set of 2-D images. This example shows you how to estimate the poses of a calibrated camera from two images, reconstruct the 3-D structure of the scene up to an unknown scale factor, and then recover the actual scale factor by detecting an object of a known size. This work shows how to reconstruct a 3-D scene from a pair 2-D images taken with a camera calibrated using the Camera Calibrator app. The algorithm consists of the following steps:

a) Match a sparse of points between the two images there are multiple ways of finding point correspondence between two images. This example detects corners in the first image using the detect MinEigenFeatures function, and tracks them into the second image using vision.PointTracker. Alternatively, we can extract Features followed by matchFeatures.

b) Estimate the fundamental matrix using estimateFundamentalMatrix.

c) Compute the motion of the camera using the cameraPose function.

d) Match a dense set of points between the two images. Re-detect the point using detect MinEigenFeatures with a reduced 'MinQuality' to get more points. Then track the dense points into the second image using vision. PointTracker.

$e$ ) Determine the 3-D locations of the matched points using triangulate.

f) Detect an object of a known size. In this scene there is a globe, whose radius is known to be $10 \mathrm{~cm}$. Use pcfitsphere to find the globe in the point cloud.

$g$ ) Recover the actual scale, resulting in a metric reconstruction.

2) Read a pair of image: First, Load a pair of images into the workspace. These images taken from the University of Agriculture Faisalabad UAF, Pakistan agricultural land with quad copter. In this time, these images are loaded in MATLAB workspace in original form (Fig. 5).

3) Undistorted image: In this step, we removed the distortion from the image, the advantage is that the image becomes clear and it is easy to find the tracking point in the given image Undistorted image shown in Fig. 6. 


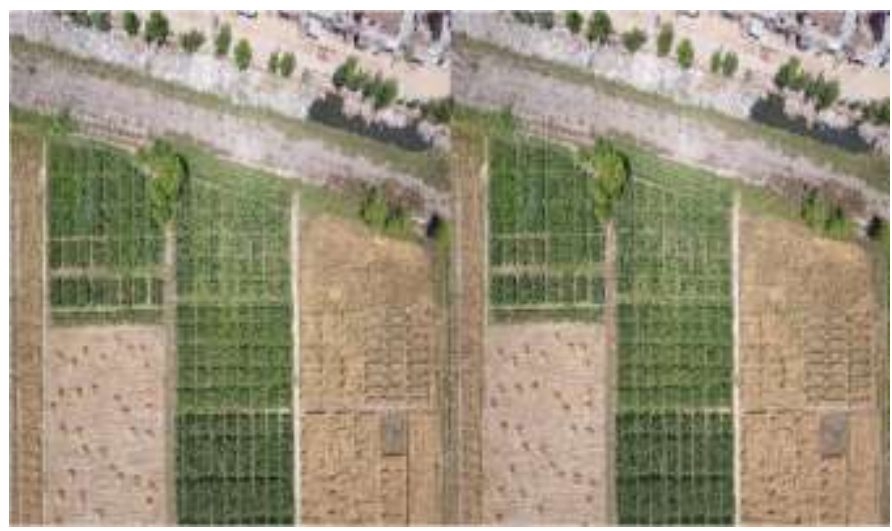

Fig. 5. Load Image Into Matlab (Sample Image Taken fom UAF Agriculture Land Site) [17].

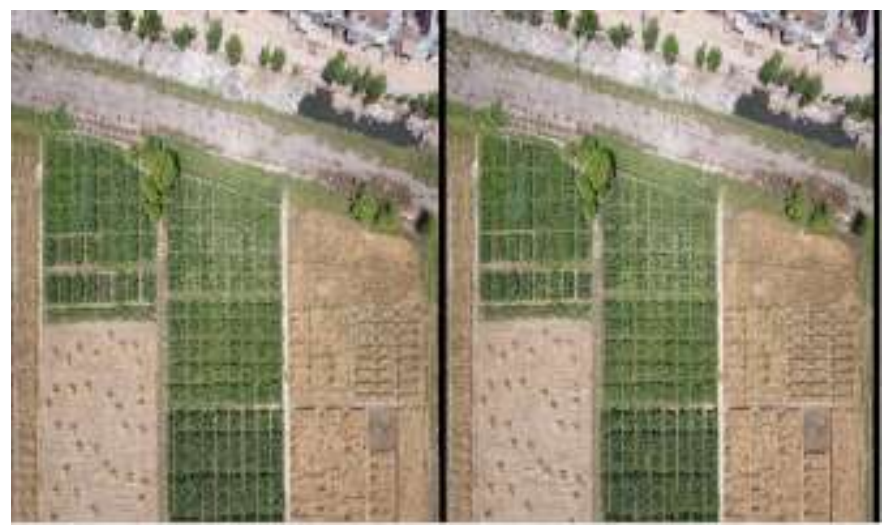

Fig. 6. Undistorted Images of Agricultural Land.

4) Track the corner point: Track out the strongest croner point from both images. These point helps to make the 3-D model. These are shown in Fig. 7 and 8.

5) Tracking feature: Match a dense set of points between the two images. Re-detect the point using detectMinEigenFeatures with a reduced 'Minquality' to get more points. Then track the dense points into the second image using vision.PointTracker. Tracking feature show in the Fig. 9.

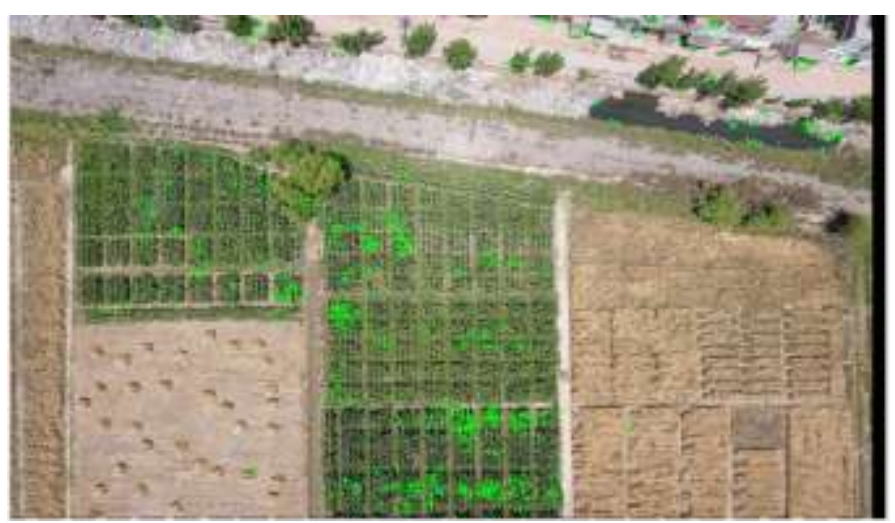

Fig. 7. Strongest 300 Corner Points from Image-1

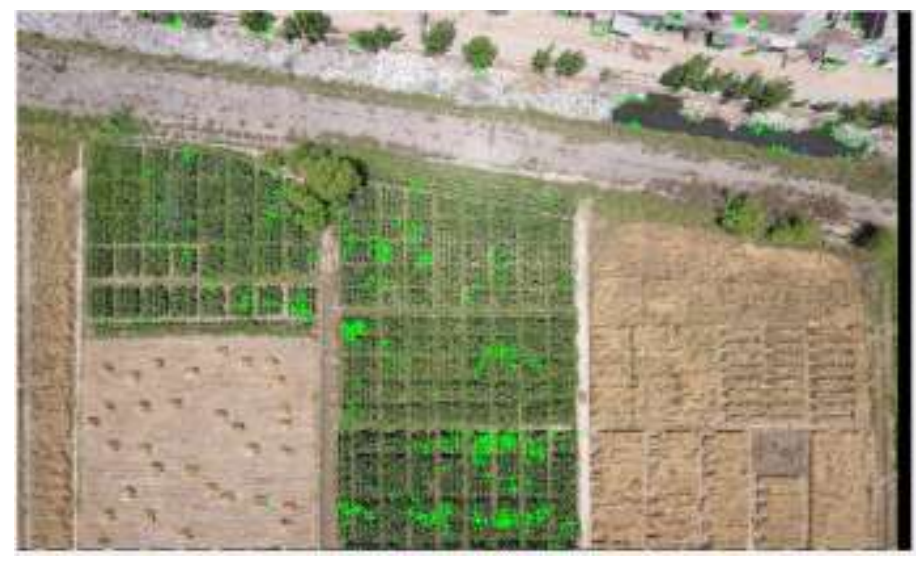

Fig. 8. Strongest 300 Corner Points from Image-2.

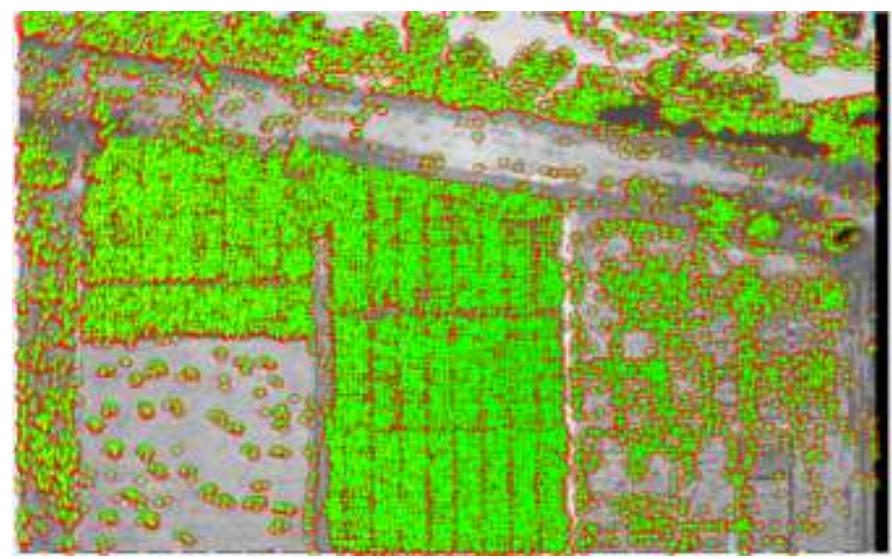

Fig. 9. Tracking Points of Both Images.

6) Epipolar inliner: Epipolar geometry is the geometry of stereo vision. When two cameras view a 3D scene from two distinct positions, there are a number of geometric relations between the $3 \mathrm{D}$ points and their projections onto the $2 \mathrm{D}$ images that lead to constraints between the image points. These relations are derived based on the assumption that the cameras can be approximated by the pinhole camera model. Track out the Epipolar inliner of image. These are shown in Fig. 10.

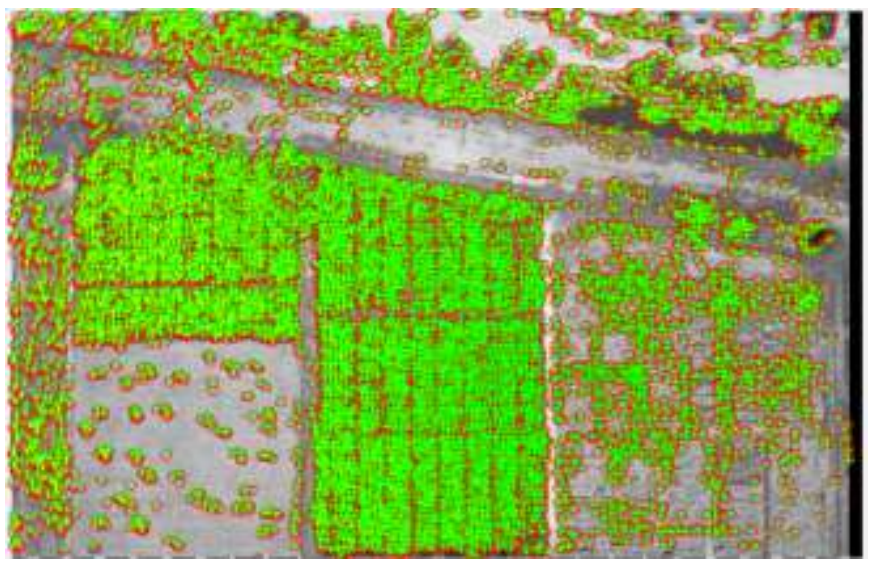

Fig. 10. Epipolar Inliner. 
7) Up to scale reconstruction of the scene: For the locations and orientations of the camera we make 3-D point cloud using pcshow function of MATLAB. Fig. 11 shows the 3-D point cloud.

8) Metric reconstruction of the scene: For Recovering the actual scale, resulting we made metric reconstruction. We can now determine the coordinates of the 3-D points in centimeters. Fig. 12 shows the metric reconstruction graph.

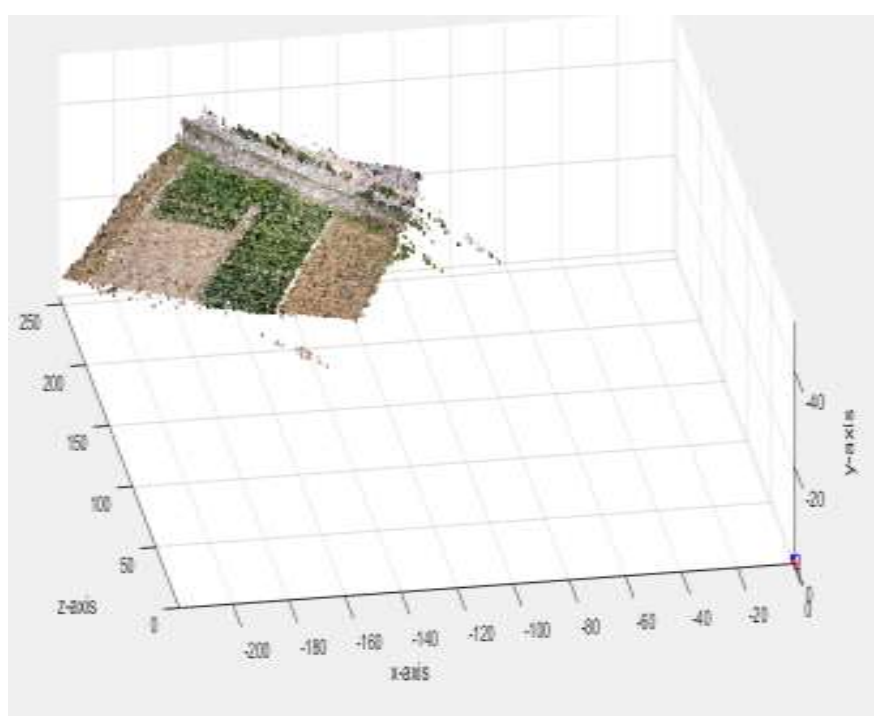

Fig. 11. 3-D Point Cloud.

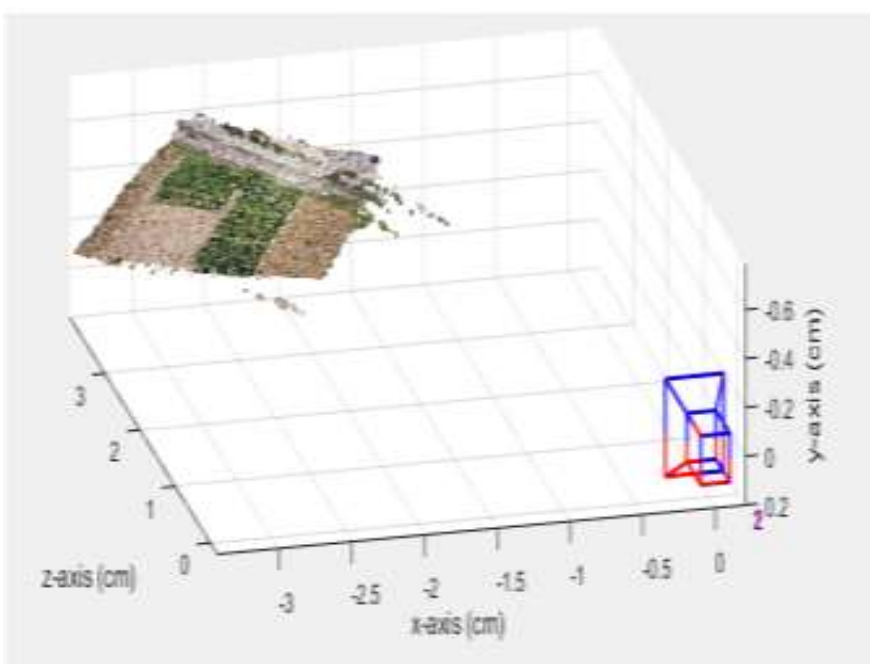

Fig. 12. Metric Reconstruction Graph.

\section{CONClusion ANd Future Work}

This paper presented a quantifiable assessment of UAS based on Structure from Motion (SfM) photogrammetry for land modeling. This showed that the UAS based land modeling and vision related approaches are suitable for capturing high resolution images at very low cost. The SfM method gave significant advantages over traditional digital photogrammetric methods. The SfM technique prevent the need of primary processing of the image like manual system testification, resolving the 3-D camera pose and samples geometric problems by using the automated algorithm for camera scene estimation. The primary results of the SfM within specific time period is drawn at related coordinate system. In this system more concentration is given for creating GCP network which provides the facilitation of transferring the absolute coordinate system data into the matric data. In this research data is collected from agriculture land with quad captor and without doing any preprocessing operation at images data. We used the SfM techniques for constructing three dimensional structures of multiple scenes. The experimental result shows that this method of construction three dimensional models is efficient and effective than other traditional approaches for agriculture land modeling. The method of UAVs photogrammetry data collection is most effective and financially suitable approach than traditional photogrammetric techniques especially in remote sensing and capturing high resolution data from high altitude. In future we extend our work by detect the diseases in agriculture crops, detecting crops patterns, and manage the all agriculture activates automatically with UAVs quad captors, flying drones, security drone by using machine learning algorithms.

\section{REFERENCES}

[1] Anders, N., Masselink, R., Keesstra, S., \& Suomalainen, J. (2013). High-res digital surface modeling using fixed-wing UAV-based photogrammetry. Proceedings of the Geomorphometry, Nanjing, China, 16-20.

[2] Dietrich, J. T. (2016). Riverscape mapping with helicopter-based Structure-from-Motion photogrammetry. Geomorphology, 252, 144157.

[3] Guarnieri, A., Vettore, A., Pirotti, F., Menenti, M., \& Marani, M. (2009). Retrieval of small-relief marsh morphology from Terrestrial Laser Scanner, optimal spatial filtering, and laser return intensity. Geomorphology, 113(1-2), 12-20.

[4] James, M. R., Robson, S., d'Oleire-Oltmanns, S., \& Niethammer, U. (2017). Optimising UAV topographic surveys processed with structurefrom-motion: Ground control quality, quantity and bundle adjustment. Geomorphology, 280, 51-66.

[5] Kanatani, K., \& Matsunaga, C. (2013). Computing internally constrained motion of 3-D sensor data for motion interpretation. Pattern Recognition, 46(6), 1700-1709.

[6] Küng, O., Strecha, C., Beyeler, A., Zufferey, J. C., Floreano, D., Fua, P., \& Gervaix, F. (2011). The accuracy of automatic photogrammetric techniques on ultra-light UAV imagery. In UAV-g 2011-Unmanned Aerial Vehicle in Geomatics (No. EPFL-CONF-168806).

[7] López, J. B., Jiménez, G. A., Romero, M. S., García, E. A., Martín, S. F., Medina, A. L., \& Guerrero, J. E. (2016). 3D modelling in archaeology: The application of Structure from Motion methods to the study of the megalithic necropolis of Panoria (Granada, Spain). Journal of Archaeological Science: Reports, 10, 495-506.

[8] Remondino, F., Barazzetti, L., Nex, F., Scaioni, M., \& Sarazzi, D. (2011). UAV photogrammetry for mapping and $3 \mathrm{~d}$ modeling-current status and future perspectives. International Archives of the Photogrammetry, Remote Sensing and Spatial Information Sciences, $38(1), \mathrm{C} 22$.

[9] Ruzgienè, B., Berteška, T., Gečyte, S., Jakubauskienè, E., \& Aksamitauskas, V. Č. (2015). The surface modelling based on UAV Photogrammetry and qualitative estimation. Measurement, 73, 619-627.

[10] Sauerbier, M., Siegrist, E., Eisenbeiss, H., \& Demir, N. (2011). The practical application of UAV-based photogrammetry under economic aspects. International Archives of the Photogrammetry, Remote Sensing and Spatial Information Sciences, 38(1), 45-50.

[11] Savvaris, A., Melega, M., \& Tsourdos, A. (2015). Advanced Surface Movement and Obstacle Detection Using Thermal Camera for UAVs. IFAC-PapersOnLine, 48(9), 43-48. 
[12] Smith, M. W., Carrivick, J. L., Hooke, J., \& Kirkby, M. J. (2014) Reconstructing flash flood magnitudes using 'Structure-from-Motion': A rapid assessment tool. Journal of hydrology, 519, 1914-1927.

[13] Turner, D., Lucieer, A., \& Watson, C. (2011, April). Development of an Unmanned Aerial Vehicle (UAV) for hyper resolution vineyard mapping based on visible, multispectral, and thermal imagery. In Proceedings of 34th international symposium on remote sensing of environment (p. 4).

[14] Watanabe, N., Nakamura, S., Liu, B., \& Wang, N. (2017). Utilization of Structure from Motion for processing CORONA satellite images: Application to mapping and interpretation of archaeological features in Liangzhu Culture, China. Archaeological Research in Asia, 11, 38-50.
[15] Westoby, M. J., Brasington, J., Glasser, N. F., Hambrey, M. J., \& Reynolds, J. M. (2012). 'Structure-from-Motion'photogrammetry: A low-cost, effective tool for geoscience applications. Geomorphology, $179,300-314$.

[16] Mathworks.com, 'Matlab for artificial intellegence', 2018. [Online]. Available:

https://www.mathworks.com/help/images/ref/checkerboard.html?search Highlight=checkerboard\&s_tid=doc_srchtitle[Accessed: 23 - Jun- 2014].

[17] University of Agriculture Faisalabad, Pakistan, 'Agriculture Land' 2018. http://oric.uaf.edu.pk/. 